\title{
The Philosophy of Quantum Field Theory
}

\author{
David John Baker \\ Department of Philosophy, University of Michigan \\ djbaker@umich.edu
}

March 12, 2015

If we divide our physical theories (somewhat artificially) into theories of matter and theories of spacetime, quantum field theory (QFT) is our most fundamental empirically successful theory of matter. As such, it has attracted increasing attention from philosophers over the past two decades, beginning to eclipse its predecessor theory of quantum mechanics (QM) in the philosophical literature. Here I survey some central philosophical puzzles about the theory's foundations.

\section{Inequivalent representations}

Perhaps the deepest new conceptual problem prompted by QFT, and certainly the one most thoroughly explored by philosophers, is the problem of inequivalent representations. ${ }^{1}$ From ordinary QM we are familiar with the use of an algebra of operators to stand for the canonical observable quantities (position and momentum, or their field-theoretic equivalents ${ }^{2}$ ). The minimal operator algebra for these canonical observables, called the algebra of observables $\mathfrak{A}$, can be represented by operators on a Hilbert space $\mathcal{H}$. This is done by assigning an operator $\pi(A)$ from the bounded operators on $\mathcal{H}$ to every operator $A \in \mathfrak{A}$. The mapping $\pi$ is called a Hilbert space representation of $\mathfrak{A}$. Given plausible assumptions, there is a unique

\footnotetext{
${ }^{1}$ To my knowledge, Huggett and Weingard (1996) were the first to discuss this problem in a published article, although Arageorgis (1995) had already explored it in some depth in his seminal dissertation.

${ }^{2}$ In bosonic field theories, the canonical observables are the field operators $\phi(x)$ and their conjugate momenta $\pi(x)$, while in fermionic theories only even polynomials of the fields and their momenta are observables.
} 
Hilbert space representation of the algebra of observables in ordinary QM. But due to QFT's infinitely many degrees of freedom, this result (the Stone-von Neumann theorem) does not hold in QFT, and there are many different representations available - continuously infinitely many. ${ }^{3}$

The problem of inequivalent representations, then, is how to understand the physical meaning of this non-uniqueness. Are the different representations different choices of notation in which to state the same physical facts? Are they distinct theories with varying physical content? Either of these options can be made to look plausible, if we focus on the right examples.

To motivate the "distinct theories" picture, first consider the fact that $\mathfrak{A}$ by itself is a rather impoverished class of physical quantities. It does not contain thermodynamic observables like temperature, for example, or the stress-energy tensor that couples to the gravitational field, or the number of particles (Ruetsche, 2002). Further quantities, or "parochial observables," can be defined only by selecting a representation $\pi$ of $\mathfrak{A}$ on a Hilbert space $\mathcal{H}$. The parochial observables are then given by self-adjoint operators on $\mathcal{H}$ which are not in the range of $\pi$. This means that different representations will have different algebras of parochial observables.

Moreover, each representation is associated with a folium, or class of algebraic states that can be represented using density operators on that representation's Hilbert space. States are assignments of expectation values to observables. In abstract algebraic terms, they are given by functionals $\omega(A)$ from observables $A \in \mathfrak{A}$ to their expectation values; relative to a representation $\pi$ on $\mathcal{H}$ these correspond to $\mathcal{H}$ 's density operators, as in ordinary quantum mechanics. Inequivalent representations have disjoint (completely non-overlapping) folia. These factors make inequivalent representations seem like distinct quantum theories, with different physical quantities and entirely different physically possible states.

Other considerations point to the opposite conclusion. A symmetry in algebraic QFT (AQFT) can be defined as an automorphism $\alpha$ of $\mathfrak{A}$ that commutes with the dynamics (i.e., with the time-evolution transformations). A symmetry of this sort acts on states as well as observables, taking $\omega$ to $\omega \circ \alpha^{-1}$. And such a symmetry can connect the folia of inequivalent

\footnotetext{
${ }^{3}$ The notion of "same representation" used here is unitary equivalence. Representations $\pi$ and $\chi$ are unitarily equivalent iff there is a one-one unitary mapping $U: \mathcal{H}_{\pi} \rightarrow \mathcal{H}_{\chi}$ such that $U^{-1} \chi(W(f)) U=$ $\pi(W(f))$.
} 
representations (in which case we say that $\alpha$ is a spontaneously broken symmetry). Normally in quantum physics, the existence of a symmetry between two sets of states is a sign of physical equivalence. So this indicates that inequivalent representations can describe physically equivalent sets of possibilities (Baker, 2011).

Early in the history of AQFT, some mathematical physicists argued that all representations of a given algebra of observables are physically equivalent. But their preferred definition of physical equivalence, weak equivalence, was deeply flawed and did not even guarantee empirical equivalence (Ruetsche, 2002; Summers, 2001). Moreover, their argument's conclusion is obviously false for physical reasons. In many cases, for example, a free (linear) theory and an interacting theory will share the same algebra of observables, with their respective states corresponding to the folia of two inequivalent representations. But (almost by definition) a free theory cannot describe the same physics as an interacting theory. It is fair to say that these weak equivalence arguments are non-starters.

Can a different definition of physical equivalence for representations succeed where weak equivalence failed? If so, this could provide a general solution to the problem of inequivalent representations. ${ }^{4}$

The most promising candidate definition is quasiequivalence (Ruetsche, 2011, 87). Two representations are quasiequivalent iff they have isomorphic algebras of parochial observables; this also means that they share the same folium. Quasiequivalent representations share the same physical quantities, and they posit all the same possible states. Consequently, it is difficult to deny that quasiequivalence is sufficient for physical equivalence.

The tougher question is whether quasiequivalence is necessary for physical equivalence. This would appear to hinge on whether folia related by a spontaneously broken symmetry are equivalent. If they are, in at least some cases, then non-quasiequivalent representations can be physically equivalent. If a spontaneously broken symmetry is always a sign of physical inequivalence, on the other hand, the way is open for quasiequivalence as a necessary condition for physical equivalence. The latter view is defended by Earman and Ruetsche (pre-print).

\footnotetext{
${ }^{4}$ Note, however, that such a definition will only involve equivalence of the physical information encoded in the uninterpreted quantum states. In an Everettian or ensemble interpretation, this will constitute physical equivalence in the fullest sense. But the same does not necessarily go for collapse or hidden-variable interpretations, which require modifying the orthodox formalism.
} 


\section{Varieties of QFT}

With the machinery of inequivalent representations on the table, we are ready to consider a perplexing problem of theory choice facing the interpreter of QFT: there is no one theory to be interpreted. In a weak sense, of course, the same is true of QM. The simple harmonic oscillator and the hydrogen atom are distinct models with their own spaces of solutions, for example. But in QM an overarching theoretical framework exists which covers all the models: the Hilbert space framework made canonical by von Neumann (1932). For QFT, there is no single canonical framework.

Rather, we are faced with two distinct research programs: algebraic QFT (AQFT), which might be called the "mathematical physicist's version" of QFT, and Lagrangian QFT (LQFT), which might be called the "laboratory physicist's version." ${ }^{5}$ On one approach to the interpretation of physical theory, this divide is not much bother. If we follow van Fraassen in asking "Under what conditions is this theory true?" (van Fraassen, 1991, 242), of course this is a question we can ask equally well of both AQFT and LQFT. The question of which theory to interpret is then just a matter of taste. But suppose we are interested, not in what the hypothetical exact truth of QFT would imply, but rather in what its mere approximate truth can tell us about the nature of our actual world? Then we must determine which of the two formalisms, AQFT or LQFT, can offer accurate answers when the two disagree. Since I prefer this latter interpretive question to van Fraassen's, I think it's well worth asking which formalism we should prefer.

To give you a rough idea of the relevant differences, AQFT is "algebraic" in the sense that the theory's basic framework assigns an algebra of observables $\mathfrak{A}(O)$ to every open region $O$ of Minkowski spacetime, whose operators $A \in \mathfrak{A}(O)$ stand for physical quantities taking on values in $O$. Each local algebra $\mathfrak{A}(O)$ is a subalgebra of the theory's global algebra of observables $\mathfrak{A}$. Parochial observables can be defined in the usual way by selecting a representation $\pi$ of $\mathfrak{A}$ on a Hilbert space $\mathcal{H}$, and states are functionals $\omega$ on $\mathfrak{A}$. Because the theory describes fields on all of Minkowski spacetime, the number of degrees of freedom is infinite (since infinitely many modes of excitation are available). As a result of this, Haag's theorem (Fraser, 2006; Earman and Fraser, 2006) implies that no interacting quantum field

\footnotetext{
${ }^{5}$ Under the moniker of AQFT, I will also include other axiomatic programs such as Wightman's, as well as constructive QFT, a research program that proceeds with the goal of constructing interacting models for rigorous axiomatic versions of the theory, including AQFT.
} 
theory exhibiting forces can be defined on the same Hilbert space representation as a free (linear) field theory. This poses a formidable obstacle to the construction of interacting theories, which has so far been achieved only for a relatively small number of idealized toy theories.

LQFT, on the other hand, succeeds in describing complicated, experimentally-confirmed interactions - but it does so only by resort to mathematically unrigorous methods. It is normally assumed, contrary to Haag's theorem, that interacting states are superpositions of free states. This, along with other false or unproven assumptions (such as the convergence of certain infinite sums in perturbation theory, and the existence of a measure for path integrals) can potentially be made rigorous sense of if renormalization group methods are taken very literally. This requires both long- and short-distance "cutoffs" - the theory is defined on a lattice spacetime of finite volume (such as a torus). ${ }^{6}$

Should LQFT or AQFT be our source for interpretive data about the relativistic quantum domain? Wallace $(2006,2011)$ argues that LQFT is the place to look for answers, while Fraser (2006, 2009, 2011) defends AQFT. Their exchange is complicated by a difference in the interpretive questions they each address. Wallace is interested in my preferred interpretive question: what QFT's approximate truth tells us about our world. Fraser, on the other hand, is concerned with whether AQFT or LQFT should qualify as QFT simpliciter, that is, as the correct theoretical framework in which to unify infinite quantum theory and special relativity (Fraser, 2009, 561-562). But along the way, Fraser does raise arguments that threaten Wallace's interpretive position.

In adjudicating their debate, the first thing to note is that a couple of Wallace's points are obviously correct, as far as it goes. First, he's right to note that no extant AQFT can describe any of the fundamental forces of the Standard Model. This means it would be absurd to draw our interpretive conclusions about QFT's domain solely from AQFT - that would imply the nonexistence of the electromagnetic field, for example. Second, Wallace rightly points out that QFT cannot be trusted to accurately describe reality outside its domain of application, in the very high-energy regimes which have not yet been experimentally investigated and where the laws of quantum gravity can be expected to apply. He sometimes

\footnotetext{
${ }^{6}$ Cutoffs are rarely posited explicitly, except as a step in infinite renormalization; 't Hooft (2007) is a notable exception. But much of the work done using LQFT can be rationally reconstructed by assuming cutoffs.
} 
suggests that this is a reason to prefer theories like LQFT which break down at high energies (Wallace, 2011, 120). But the upshot is rather that whatever version of QFT we interpret, we should not trust its implications within the high-energy domain. By analogy, we trust Newtonian mechanics only in the low-speed domain where it approximates relativity, but this is no reason to prefer a theory that breaks down as objects approach the speed of light. Still, Wallace is right that when standard axioms for AQFT assign observables to every arbitrarily small region, this is not necessarily a virtue. ${ }^{7}$

Wallace is correct that, to at least some interpretive questions (e.g., is there a photon field that interacts with an electron field?), LQFT provides the obvious right answer and presentday AQFT cannot. Should we therefore take LQFT to be the arbiter of all interpretive questions in QFT's domain? LQFT involves idealizations of its own. The most significant one is the long-distance cutoff. Cosmological theory and astronomical observations strongly suggest that our universe is spatially infinite. But remove the long-distance cutoff and LQFT starts to look much more like AQFT. Inequivalent representations become relevant, and in particular, the premises of Haag's theorem are satisfied (Fraser, 2006, 63-66). So AQFT will be required in order to answer the interpretive questions raised by inequivalent representations - questions about the nature of physical equivalence, spontaneous symmetry breaking (at least for non-gauge symmetries), superselection rules and charges, and many other problems of the sort discussed in Ruetsche $(2002,2011) .{ }^{8}$ On the other hand, we must obviously trust LQFT when it comes to questions about the specific nature of the three Standard Model forces.

I submit that the controversy between LQFT and AQFT is really a question about the domains of application of the two theories. Ideally, if either theory were fully satisfactory, it would provide a good approximation to reality in all domains where the influence of gravity is negligible. But neither is satisfactory - each involves idealizations, either concerning the nature of spacetime and the number of degrees of freedom (as in LQFT) or concerning the nature of forces (in present-day AQFT). So LQFT (resp., AQFT) should be trusted in those

\footnotetext{
${ }^{7}$ Not all work in AQFT assumes that observables are well-defined at short distances; perturbative AQFT drops this assumption (Buchholz and Verch, 1995).

${ }^{8}$ Given that QFT cannot be trusted to accurately describe arbitrarily short distance scales, it seems best not to put much trust in (non-perturbative) AQFT's description of locally inequivalent representations, since local inequivalence arises from the infinity of arbitrarily high-energy modes of the field. Globally inequivalent representations, on the other hand, would appear to be well-motivated physically.
} 
domains of inquiry where its own idealizations are unproblematic and where the idealizations of AQFT (LQFT) are physically unrealistic. Rather than a global debate that can be won or lost over all of the domain QFT aspires to describe, this is a debate that must be waged separately for each interpretive question. For the remainder of this essay I will consider questions that I think lie within the proper domain of AQFT.

\section{$3 \quad$ Particle interpretations}

QFT is the central tool of what is called elementary particle physics. The name would seem to imply that the theory posits a reality consisting fundamentally of indivisible particles. There are a few guises under which this implication can be precisified; under all its precisifications, it has been the target of powerful arguments. These arguments are often said to refute "particle interpretations" of QFT, but this term can mean a couple of different things.

In particular, by a particle interpretation, we might mean:

- a fundamental particle interpretation, on which particles are among the physically fundamental entities, or

- a non-fundamental particle interpretation, on which particles exist, although only as composite or non-fundamental entities.

Moreover, there is an ambiguity in the term particle itself. It has been taken to denote:

- localizable particles, whose fundamental properties include position, or

- quanta, which are countable but not necessarily localizable.

Existing arguments against particle interpretations are usually clear about whether they target localizable or quanta interpretations, but less clear about whether they extend to non-fundamental as well as fundamental interpretations. I hope to clarify this in what follows.

The arguments against localizable particle interpretations are the most decisive. ${ }^{9}$ These proceed by establishing the nonexistence of a position observable meeting certain localitymotivated conditions. Malament (1996) proves the nonexistence of a projection-valued

\footnotetext{
${ }^{9}$ It is, however, important to keep in mind their limitations. In particular, like all the work surveyed in this article, they are confined to the orthodox quantum formalism and thus cannot rule out particle interpretations which depart from this formalism, such as hidden-variable interpretations (Rachiele, in progress).
} 
measure for position in relativistic quantum mechanics (which, by contrast, exists in nonrelativistic QM). While this implies the nonexistence of a self-adjoint position operator, it is commonly thought that more general positive operator-valued measures (POVMs) can also represent measurable quantities. But Halvorson and Clifton (2002) show further that there is no POVM for position in relativistic quantum mechanics, and that operators describing the number of particles in a region in QFT also do not exist. These arguments appear to establish that the sort of things we ordinarily call "particles" (leptons, quarks, gauge bosons), whether fundamental or not, are not localizable. The best hope for resisting these arguments is probably the Newton-Wigner localization scheme (Fleming, 2000), but this has been the target of powerful objections (Halvorson, 2001).

Quanta interpretations are logically weaker than localizable particle interpretations, so one might expect them to fare better. And indeed, while there are significant arguments against quanta, their soundness is more debatable. These fall into two classes: arguments from inequivalent representations and arguments from interactions.

In their original form, arguments from inequivalent representations proceed in the following way (Halvorson and Clifton, 2001; Wald, 1994). First, we note that the difference between some pairs of inequivalent representations appears to be a matter of perspective. In the most often-cited example, the so-called Rindler or Fulling representation of $\mathfrak{A}$ seems to encode the perspective of an observer accelerating at a constant rate, while the Minkowski representation corresponds to an inertial observer. In particular, the two representations have different parochial observables and different folia of states. Intuitively, the parochial observables for the Rindler (resp. Minkowski) representation correspond to quantities measurable by an accelerating (inertial) observer, while the vacuum state of the Rindler (Minkowski) representation's folium corresponds to the accelerating (inertial) observer's definition of the vacuum.

Now, among the parochial observables for each representation is a number operator encoding predictions about the total number of particles in the universe. With a creative application of AQFT's mathematical tools, we can determine the expectation value of the Rindler representation's number operator in the Minkowski vacuum state (Halvorson and Clifton, 2001, 448-452). Intuitively, this tells us how many particles an accelerating observer will ascribe to the state that an inertial observer would call empty of particles. Since this expectation value is infinite, it appears that the accelerating and inertial observers have dif- 
ferent definitions of 'particle,' and by the accelerating observer's definition, the supposedly Minkowski vacuum is full of particles. Since there is nothing physically privileged about either observer's definition, it must be that the number of particles is a perspective-dependent fact. If particles belonged to QFT's fundamental ontology, the number of particles would not be dependent on one's perspective - here we assume, plausibly, that the number of fundamental entities in the universe is an objective fact. So if this argument succeeds, it rules out fundamental particle interpretations.

Arageorgis et al. (2003) have criticized this argument on both philosophical and formal grounds. On the philosophical side, they argue that it does not establish "that particles are not 'real,' any more than the need to relativize the electric and magnetic fields to a reference frame would show that these fields are not real." (186-187) This objection does not undermine the argument, though, if we understand it as an argument against fundamental particle interpretations. After all, the non-invariance of the electric and magnetic fields does imply that they are not fundamental; the invariant field tensor is the fundamental quantity in electrodynamics.

Formally, Arageorgis et al. object to Halvorson and Clifton's calculation of the expectation value for the Rindler number operator in the Minkowski vacuum. They note that there are many Fock representations of the algebra of observables inequivalent to the Minkowski representation, and any of these representations possesses a number operator which will likewise register an infinite expectation value for the Minkowski vacuum by Halvorson and Clifton's lights (191-192). They conclude that Halvorson and Clifton's calculation is either a merely formal trick, or it has the absurd consequence that the Minkowski vacuum is full of many different varieties of particle, one for each inequivalent Fock representation.

But this does not follow. In the case of the Rindler representation, we have an argument from physical principles that this representation's definition of 'particle' corresponds to an (accelerating) observer's particle concept. No such argument has been supplied for any of the other inequivalent Fock representations. Were we supplied with such an argument, we would have every reason to accept that the relevant observer(s) would ascribe infinite particle content to the Minkowski vacuum. In the absence of such an argument, there is no reason to ascribe any physical meaning to any of these representations' attendant number operators.

Even Arageorgis et al. grant, however, that related arguments from curved spacetime succeed against particle interpretations. To construct a Fock space representation, a family of 
timelike isometries of spacetime is needed in order to define the single-particle Hilbert space. Some spacetimes, such as Schwarzschild spacetime, possess more than one family of timelike isometries. These will each correspond to a different, inequivalent Fock representation, none physically privileged over the others and each with its own definition of particle. This undermines fundamental particle interpretations in much the same way as the previous argument. Moreover, some spacetimes (such as expanding Friedmann-Robertson-Walker models) have no timelike isometry group and hence no Fock representation. It is at best unclear whether any definition of particle can apply to QFTs on such spacetimes (Ruetsche, 2011, 256-259). If none does, we would appear to have an argument against even nonfundamental particle interpretations.

Arguments from interactions also attack non-fundamental as well as fundamental particle interpretations. Fraser (2008) has argued against the existence of particles in interacting QFTs constructed within the AQFT paradigm. Fraser begins by arguing that QFT cannot describe particles unless it possesses a Fock space representation. Fock representations alone include a particle number operator (whose expectation value gives statistical predictions for the number of particles in the universe) and a unique vacuum state which can be interpreted as the zero-particle state. Fraser then shows that interacting QFTs cannot possess Fock representations, making a particle interpretation of these QFTs impossible.

Her argument proceeds as follows. Thanks to Haag's theorem, interacting theories cannot share a Hilbert space representation in common with the free theory. So we cannot interpret an interacting state as describing superpositions of free particles (Fraser, 2008, 14-20). A more tempting option is to construct a different Fock representation unique to the interacting theory. But Fraser goes on to show that this option is unavailable. In an interacting theory, there is no covariant way to divide the single-particle wavefunctions into positiveand negative-frequency classes - a crucial step in the construction of a single-particle Hilbert space. As a result, there is no way to define a one-particle space that can be used to construct a Fock representation (Fraser, 2008, 20-23). And with no Fock representation, there is no way to interpret a state of an interacting QFT as containing quanta.

If Fraser's argument succeeds, in interacting QFT there is no physical quantity corresponding to the number of particles. Since her argument nowhere assumes that such a quantity must be fundamental, we have another argument against even non-fundamental particle interpretations. Several attempts to make sense of arguments against particles in 
QFT have focused on the hope that particles exist as derivative entities - for example, as excitations of the field (Halvorson and Clifton, 2001, 460). But it appears that in interacting theories, there are no countable particles, even if we allow that they might be composite entities. How, then, are we to make sense of the fact that the entities detected by cloud chambers and collider detectors are treated by physicists as countable particles?

I submit that working physicists' apparent ontological commitment to particles should be understood as an approximation to the truth. In physics it's common for a less-fundamental theory to approximate a more fundamental theory in some limited domain (normally a proper subset of the physical situations in which the more fundamental theory applies). This is the case in QFT as well.

Although its quantitative predictions are never completely accurate, free QFT provides a good approximation to interacting QFT in the so-called asymptotic scattering limit, before and after scattering interactions (see Bain, 2000). Since free states can be understood as particle states, we have a theory with an ontology of particles which approximates a more fundamental theory without a particle ontology. So under the working assumption that interacting QFT is exactly true, "particles exist" may be approximately true, even though this statement is strictly speaking false. Given that interacting QFT is not itself our final, most fundamental theory, perhaps we should then say that its ontology provides a better approximation to the truth than the particle ontology of free QFT on Fock space, although the latter is also a good approximation in some domains. This, I suggest, is the best way to maintain something like the pre-philosophical conception of high-energy particle physics while accepting arguments against quanta.

\section{$4 \quad$ Field interpretations}

The natural alternative to a particle interpretation is a field interpretation, according to which the theory's ontology is in some sense a quantum version of a classical relativistic field. Again, there are broad and narrow varieties. The broadest notion of a field interpretation is very broad indeed. In contrast with particle interpretations, it involves simply the claim that the fundamental entities are neither localizable nor countable, but rather that matter in QFT consists of properties of spacetime points or regions, or something similar. I know of no general argument against this sort of broad field interpretation. 
The narrower notion of field interpretation is another matter. This class of views, sometimes referred to as wavefunctional interpretations, hold that QFT is a field theory in the same sense that QM is a particle theory. Its states are superpositions of classical field configurations, just as QM states are superpositions of classical particle configurations. The natural way to represent this mathematically is with a functional $\Psi(\varphi)$ which assigns complex amplitudes to each state $\varphi$ of the classical field. The expectation values of the field operators $\phi(x)$ are then interpreted as the mean value of the field in a given superposition $\Psi(\varphi)$.

In Baker (2009) I present two arguments against wavefunctional interpretations, one of which rests on symmetry breaking and the other on interactions. I will focus here on the argument from symmetry breaking, because I now have more to say about it. The argument proceeds by noting that, as discussed in Section 1, broken symmetry transformations can relate the folia of inequivalent representations. In the case of broken spacetime symmetries, it is implausible to suppose that any two such folia are physically inequivalent, since spacetime symmetries typically amount to mere changes in coordinates (under the passive interpretation). But adherents of the wavefunctional interpretation cannot allow that two such folia are physically equivalent, since broken symmetry transformations will not leave the field operators unchanged.

I see some room, though, for followers of the wavefunctional interpretation to deny this last step in the argument. As Baker (2011) argues, it is possible for mathematically distinct operators to stand for the same physical quantity if they are related by a symmetry. This amounts to a change in which mathematical entities represent which physical quantities. It is possible that, by maintaining that formally different field operators can stand for the same physical field quantity, adherents of the wavefunctional interpretation can preserve their view in the face of the symmetry breaking argument in Baker (2009).

Moving beyond wavefunctional interpretations, let's consider some possibilities for field interpretations in the broad sense. The oldest proposal is due to Teller $(1995,2002)$. It posits an ontology of propensities for the system to exhibit properties upon measurement. These propensities are represented by the expectation values of products of the field operators. Thus, if the symmetry breaking argument against the wavefunctional interpretation succeeds, it will also count against Teller's interpretation. Huggett and Weingard (1996) criticize this interpretation, arguing that its ontology of brute propensities is unsatisfactory. 
More recently, Wallace and Timpson (2010) have advanced an ontology for QFT that fits under the heading of broad field interpretations. On their view, spacetime state realism, there is a fundamental quantity assigned to each region of spacetime, represented by the local state of that region. For example, in the algebraic approach every open region $O$ is associated with a subalgebra $\mathfrak{A}(O)$ of the algebra of observables $\mathfrak{A}$, whose elements stand for the quantities localized in that region. The restriction of the global quantum state $\omega$ to $O$ is then a state of $\mathfrak{A}(O)$, which we call the local state $\omega_{O}$. On Wallace and Timpson's view, rather than just codifying the expectation values of physical quantities, the state $\omega_{O}$ itself represents a fundamental property of region $O$. In effect, QFT describes a "field" of local states in much the same way that electrostatics describes a field of vectors.

This view has much to recommend it, but a couple of criticisms have been raised. First, the connection between the fundamental quantities represented by the local states and the theory's predictions is somewhat opaque. We derive statistical predictions from the quantum state via expectation values, but what is the meaning of an expectation value of energy, for example, if energy is not among the fundamental quantities (which are exhausted by the local states)? Moreover, how are we to make sense of a deterministic theory whose fundamental quantities appear to be specified indirectly by way of statistical expectation values? ${ }^{10}$ Additionally, Ismael and Schaffer (forthcoming) accuse the state realist of positing a great deal of surplus structure, since the state realist cannot do without the global state $\omega$, and all the physical information contained in the local states is uniquely specified by the global state.

A somewhat similar field interpretation has been advanced by Deutsch and Hayden (2000) and defended by Arntzenius (2012), who dubs it Heisenberg operator realism. The picture involves a modification of the ordinary Heisenberg-picture formalism. In the Heisenberg picture, observables are assigned to locations in time as well as space (so that e.g. "the total energy at 2:00" is treated as a different observable from "the total energy at 3:00"). The state is treated as unchanging (or more accurately, it encodes the physical state of all of spacetime), but it is physically contingent-different states correspond to different assignments of expectation values to the observables (which are held fixed). In Heisenberg operator realism, on the other hand, the state is treated as physically necessary and the observables as contingent. The interesting thing about this formalism is that the algebras of

\footnotetext{
${ }^{10}$ Lewis (2013) and Baker (2014) both make this point.
} 
observables assigned to spacetime regions are entirely local and separable entities. And there is no obstacle in principle to treating these "observables" as the values of operator-valued field quantities. As a result, if the "state" is treated as an aspect of the laws rather than a concrete thing (making it something akin to a density operator-valued physical constant), this field interpretation would appear to ascribe a local, separable ontology to QFT.

It seems too good to be true, and indeed, Wallace and Timpson (2007) argue that Deutsch and Hayden's formalism misrepresents their interpretation. Wallace and Timpson identify a class of space-and-time-dependent phase transformations on the observables which leave their expectation values unchanged. These transformations, they argue, should be treated as gauge symmetries, since they vary locally in their effect on the observables and don't alter anything of physical interest. So the real physical content of the theory is given by equivalence classes of the Heisenberg observables under these transformations. And these equivalence classes are isomorphic to the local states described in Wallace and Timpson (2010) - making Heisenberg operator realism a mere notational variant of spacetime state realism. Arntzenius $(2012,116-120)$ criticizes this argument on the grounds that Wallace and Timpson's transformations do not deserve to be called symmetries, because they fail to leave the theory's dynamical laws unchanged. After all, not every transformation that preserves a theory's empirical content is a symmetry. Symmetries must also preserve the laws.

However this debate is resolved, to my mind the following brief remark can form the core of a major objection to Heisenberg operator realism: "[I]n a physical theory we would normally prefer that what is observable (i.e., the expectation values derived from [the state] would emerge from a physical analysis of measurement, rather than by fiat)" (Wallace and Timpson, 2007, 954). Unlike hidden-variable or collapse interpretations, for example, Heisenberg operator realism gives no physical story about how measurements (or other macroscopic phenomena, for that matter) are to be explained by the underlying structure of observablevalued fields. At least, there is no story beyond the brute posit that such and such a distribution of operators corresponds to such and such a set of statistical expectation values. Quite an unsatisfying form of explanation! But as we saw above, essentially the same objection applies equally to Wallace and Timpson's own state realist interpretation. It appears that a perspicuous explanation for the expectation values is a major missing piece in extant field interpretations. 


\section{Coalesced structures}

Throughout the above discussion, I have used tools and concepts familiar from interpretive work on previous physical theories. But it could be that the interpretation of QFT cannot succeed without new tools and concepts. As we've seen, the problem of inequivalent representations in particular seems to resist traditional interpretive techniques. Ruetsche (2011) has suggested that any successful interpretation of QFT will have to depart from traditional conceptions of physical law and possibility. She proposes an alternative conception called the coalesced structures view.

Ruetsche's view is best understood by looking at the considerations she marshals to motivate it. An important example for her purposes is given by QFT on curved spacetime (Ruetsche, 2011, 237-239). For a given QFT, in some applications we may wish to treat the quantum field as a source for the gravitational field, in which case we will need to know the expectation value of the stress-energy tensor. This expectation value is guaranteed to exist if we require that all states meet the so-called Hadamard condition. This requirement amounts, on Ruetsche's view, to the stipulation that Hadamard states are the only physically possible states for present purposes. But with different applications in mind, we might want to derive the emission of radiation from an evaporating black hole. This requires non-Hadamard states, which are then presumably to be counted as physically possible.

This example has two crucial features of significance to Ruetsche. First, although the same QFT is being employed in both the gravitation and black hole applications, the two

applications seem to require two different sets of physically possible states. Second, they also employ different physical quantities. For example, the stress-energy tensor is a crucial observable in the gravitation application, but the vacuum state employed in the black hole application is outside the domain of this observable.

Traditionally, interpreters of physics have assumed that states break down into two sharply-defined classes: the physically possible ones allowed by the laws, and the physically impossible ones. This picture cannot be sustained, Ruetsche suggests, in the face of examples like QFT in curved spacetime, where a state can count as physically possible in one application of the theory but impossible in another. She suggests replacing the traditional picture with her coalescence view, according to which physical possibility comes in degrees. The full algebraic state space of the algebra of observables $\mathfrak{A}$ provides what Ruetsche calls 
the "primordial" possibilities, or the worlds physically possible in the broadest sense. This space delimits the full "scope of the theory." (Ruetsche, 2011, 289) The observables in $\mathfrak{A}$ are the physical quantities that all these states have in common. But for a given application, we might restrict our attention to states possible in a narrower sense (for example, Hadamard states). This may allow us to incorporate further physical quantities outside $\mathfrak{A}$ (such as the stress-energy) in our explanations.

In evaluating Ruetsche's view, we must carefully gauge what is (and is not) accomplished by her core postulate that possibility comes in degrees. She argues: "Identifying physical possibility with primordial possibility, period, we undermine the nomic force of many explanations encountered in physical practice - explanations mediated by laws which hold only of a proper subset of primordial possibilities." (Ruetsche, 2011, 290) In a sense, the same is true of many explanations elsewhere in physics, besides QFT. For example, it is not normally treated as a law of electrodynamics that retarded electromagnetic waves are emitted from radio antennae, but advanced waves do not converge on antennae and become absorbed. But we normally assume, for broadly causal reasons, that the former process and not the latter is typically found in nature. Does this undermine the nomic force of electromagnetic explanations for the behavior of radio antennas and waves? Only if such explanations are meant to apply with the force of nomic necessity, which is at best a debatable assumption. Similar things could be said about explanations in thermodynamics that appeal to a low-entropy past. $^{11}$

I also question Ruetsche's identification of the primordial possibilities with the complete set of algebraic states of $\mathfrak{A}$. This set will typically include free states, as well as states for any possible interacting system that has $\mathfrak{A}$ as its algebra of observables. For example, the free boson field shares its algebra of observables with the $\phi^{4}$ interacting theory - and with all other QFTs of polynomial scalar self-interactions. But surely it is a physically necessary fact, even in the broadest sense of physical necessity, that there are forces. So to suggest that a collection of states including both free and interacting states should be lumped together as belonging to the "same theory" is quite a stretch. It is true that many important physical explanations - the explanation for the Hawking effect, for example - use free QFT. But it

\footnotetext{
${ }^{11}$ On the other hand, if one is convinced that the postulates supporting these electromagnetic and thermodynamic explanations are laws, and that it is important in other explanatory contexts (for example, ones appealing to time-reversal symmetry) to suspend these postulates, then coalescence is everywhere in physics. Ruetsche's approach is then essential to understanding many other theories besides QFT.
} 
seems patently obvious that this is an idealization. We pretend that there are no forces because the detailed nature of the forces does not matter to the relevant explanation and it would be too difficult to derive the predictions if we filled in such details. But this is a far cry from positing that the laws of nature permit free particles to exist.

A less radical alternative view can, I think, replicate many of the successes of coalesced structures. I have in mind a view that Ruetsche has called tempered universalism. On this view, the traditional picture of physical possibility is retained, and a "large" representation of $\mathfrak{A}$ (in the sense that it is a direct sum of many irreducible representations) is used to stand for the physical quantities. The states of the large representation's irreducible subrepresentations stand for the physical possibilities. So in an application requiring inequivalent representations, like spontaneous symmetry breaking, this view would take each ground state to be physically possible in the same sense, but also recognize the global magnetization observable of each representation as physically significant. This seems rather more straightforward than the coalesced structure theorist's view that the actual ground state's magnetization observable is physically significant only relative to this particular application, and the other ground states are in some sense "less possible" than the actual one.

Ruetsche has criticized this sort of view by arguing that it introduces surplus theoretical structure, in the form of too many observables. Consider any two inequivalent, irreducible subrepresentations of the large representation. The parochial observables native to one subrepresentation will provide no physical information about the states of the other subrepresentation's folium. So these parochial observables play no role in describing the vast majority of the theory's states. And since every irreducible subrepresentation has its own family of parochial observables, that is a lot of surplus structure!

I am not convinced, however, that the sort of superfluousness Ruetsche sees in the parochial observables is particularly disturbing, or without precedent in past theories. To cite one such precedent, the distribution of charge is an important quantity in electrodynamics, but it provides no non-trivial physical information about the (continuously infinitely many) vacuum solutions. A clearer case may be classical particle mechanics. The spatial distance between particles $A$ and $B$ is a significant quantity, but it does not take on a value in solutions where $A$ and $B$ don't exist. Ruetsche's objection amounts to the observation that, on a universalist-style view, physically contingent facts have a significant role in determining which physical quantities take on values, or provide physically significant information about 
a state. But on reflection, this seems unobjectionable and hardly novel.

The coalescence view retains at least one significant potential advantage over the tempered universalist view I've advocated. Understood permissively enough, Ruetsche's view allows that in some applications, a theory's predictive content might coalesce to a representation of $\mathfrak{A}$ and a state from a different representation's folium. ${ }^{12}$ For example, as in Halvorson and Clifton's argument against particles, we might be interested in the value assigned to some physical quantities of the Rindler representation by the Minkowski representation's vacuum state. Since the Rindler vacuum is probably not a physically possible state (since it cannot be extended to a Hadamard state on all of spacetime), the Rindler representation will not be a subrepresentation of the large representation posited by the tempered universalist. So this sort of application would appear to make no sense for the tempered universalist, while making perfect sense on the coalescence view. Unless we want to rule out this sort of application altogether, the coalescence view still seems to explain more of the actual usefulness of QFT than any alternative I'm aware of. The upshot may be that coalescence gives an accurate description of some of QFT's more unusual applications, but is unnecessary for understanding most applications of the theory, which fit comfortably within the tempered universalist picture.

\section{Spacetime and QFT: the CPT theorem}

By and large, Minkowski spacetime remains the same in QFT as it was in classical theories. But there is some novelty to be found in the theory's description of matter's relationship with spacetime. At least, such novelty is suggested by the CPT theorem, a result that purports to relate the discrete symmetries of spacetime to the charge conjugation transformation that exchanges matter with antimatter. The theorem is usually stated as follows: if $C$ is charge conjugation, $P$ is the parity transformation that switches left and right, and $T$ is the time-reversal transformation, the combination of the three (CPT) is a symmetry of any Lorentz-invariant QFT.

The apparent physical moral — that the possibility of a preferred temporal and/or spatial orientation to spacetime is not independent of a "charge orientation" picking out a preferred definition of positive charge - can appear quite puzzling. As Greaves (2010, 29) points out,

\footnotetext{
${ }^{12}$ Indeed, Ruetsche (personal communication) has in mind this permissive form of coalescence.
} 
it seems like a necessary connection between distinct existences. What do handedness, charge, and the direction of time have to do with each other? (The link between PT, a spatiotemporal transformation, and $\mathrm{C}$, which only acts on non-spatiotemporal quantities, is especially mysterious.) Moreover, how can facts about a theory's continuous symmetries (Lorentz invariance) entail facts about discrete symmetries like CPT? In the remainder of this section I will examine a few answers to Greaves's puzzle.

Greaves (2010) attempts to illuminate her puzzle by considering a classical analog of the CPT theorem. The classical version is a PT theorem which applies to polynomial tensor field theories. Greaves argues that properly understood, time-reversal should reverse charge as well, and so what is in reality a PT theorem may have been misunderstood, in the context of QFT, as a CPT theorem. She then shows that the classical PT theorem has a clear physical explanation: Lorentz-invariant tensor fields are incapable of privileging a temporal orientation (a preferred definition of past and future), but they are capable of privileging a spatiotemporal orientation - the four-dimensional equivalent of a preferred handedness, or a preferred pair of a temporal direction and a spatial orientation. So the PT theorem is explained by the properties of tensors.

Swanson (preprint) criticizes this approach, arguing that the resources of quantum theory are required to understand the physical explanation for CPT invariance. He begins with an exposition of the CPT theorem in AQFT. Remember that in AQFT, the quantities that take on values in a region of spacetime form a "local" algebra of observables. Each such algebra has a noncommutative product defined on it (the product between operators), which can be split into two components, a Lie product and a Jordan product. The Lie product is the one whose structure determines the link between continuous spacetime symmetries and the observables that generate them (e.g., between momentum and spatial translation symmetry, or between the Hamiltonian and time translation).

The algebraic CPT theorem arises from the provable existence of a transformation $\Theta$ of the whole (global) algebra of observables which reverses the order of the Lie product (taking the product of $A$ with $B$ to the product of $B$ with $A$ ) and meets the criteria for a symmetry. Because it reverses the order of the Lie product, one can prove (via the Lie product's role in structuring the continuous spacetime symmetries) that $\Theta$ reverses both spatial and temporal orientation (implementing PT). But the Lie product has another physically significant role in the theory: it appears in the definition of charge conjugation. As a result, $\Theta$ must also 
have the effect of conjugating charges. So we have an explanation for the CPT theorem: the same piece of theoretical structure that determines the spatiotemporal orientation in AQFT also has the role of determining the difference between positive and negative charge. This is Swanson's answer to Greaves's puzzle. Because the quantum-mechanical structure of AQFT's algebras of observables is essential to his answer, Swanson claims it is a mistake to look to classical PT theorems for a physical understanding of CPT.

The recent proof of Greaves and Thomas (2014), on the other hand, suggests that CPT invariance is not essentially a quantum phenomenon. Rather, it applies equally in classical theories; hence, the physical explanation would seem to involve concepts common to all field theories, rather than anything specific to QFT. Greaves and Thomas prove a version of the CPT theorem for what they call "formal field theories"-polynomial functions of field variables, which may be naturally interpreted as equations of motion or Lagrangian or Hamiltonian densities for Lorentz-invariant field theories. The idea is that a formal field theory specifies what is left when one abstracts away from a theory's state space framework (Lagrangian versus Hamiltonian) and its status as either quantum or classical. Greaves and Thomas prove that any formal field theory meeting certain well-motivated formal conditions is CPT invariant. Along the way they show that a PT theorem for spinor field theories is not in the offing; this would appear to mean that the purely geometric understanding of CPT earlier proposed by Greaves (2010) does not apply to spinor fields like the electron.

The Greaves-Thomas CPT theorem is a great accomplishment, especially given its generality in applying to classical as well as quantum theories. I am puzzled, though, by their claim to have proven it "within the framework of mainstream (Lagrangian) quantum field theory" (Greaves and Thomas, 2014, 46). Their framework of formal field theories assumes that these theories are Lorentz invariant; but as we saw above in Section 2, the only known way to work rigorously with Lagrangian QFT is to impose a minimum length cutoff by putting the theory on a lattice. But this breaks Lorentz invariance, since the lattice spacing is not invariant under Lorentz boosts. So no known rigorous version of LQFT will count as a formal field theory. As it stands, the only existing quantum theories that count as formal field theories are free theories and certain AQFTs, so their theorem is only known to apply to these theories. ${ }^{13}$ Greaves and Thomas claim that their theorem makes contact with working

\footnotetext{
${ }^{13}$ Since there exist AQFTs with non-polynomial interactions or with no associated Lagrangian, the Greaves-Thomas will apply to some but not all AQFTs.
} 
physicists' picture of QFT in a way that the algebraic CPT theorem does not, but I see no reason to accept this claim in the absence of rigorous, Lorentz-invariant LQFTs.

We seem to be confronted with a second puzzle about CPT. If Swanson's argument succeeds, the CPT theorem is essentially a quantum result, and any physical understanding of it will require the resources of QFT. On the other hand, the Greaves-Thomas theorem seems to point toward a common explanation for CPT invariance in classical and quantum field theories. A deeper comparison of the Greaves-Thomas theorem with algebraic versions of the CPT theorem is needed if we are to adjudicate this dispute.

\section{Acknowledgements}

Thanks to Joshua Hunt, Charles Sebens and Noel Swanson for comments on a previous draft, and to Laura Ruetsche for helpful discussions.

\section{References}

Arageorgis, Aristidis (1995), Fields, Particles and Curvature, University of Pittsburgh: unpublished dissertation.

Arageorgis, Aristidis, John Earman and Laura Ruetsche (2003), "Fulling Non-uniqueness and the Unruh Effect: A Primer on Some Aspects of Quantum Field Theory," Philosophy of Science 70:164-202.

Arntzenius, Frank (2012), Space, Time, and Stuff, Oxford: Oxford UP.

Bain, Jonathan (2000), "Against Particle/Field Duality: Asymptotic Particle States and Interpolating Fields in Interacting QFT," Erkenntnis 53:375-406.

Baker, David John (2009), "Against Field Interpretations of Quantum Field Theory,” British Journal for the Philosophy of Science 60:585-609.

Baker, David John (2011), "Broken Symmetry and Spacetime," Philosophy of Science 78:128-148.

Baker, David John (2014), "Review: Frank Arntzenius, Space, Time, and Stuff," Philosophy of Science 81:171-174.

Buchholz, Detlev and Rainer Verch (1995), "Scaling algebras and renormalization group in algebraic quantum field theory," Reviews in Mathematical Physics 7:1195.

Deutsch, David and Patrick Hayden (2000), "Information Flow in Entangled Quantum Systems," . URL http://arxiv.org/abs/quant-ph/9906007 
Earman, John and Doreen Fraser (2006), "Haag's Theorem and its implications for the foundations of quantum field theory," Erkenntnis 64:305-344.

Earman, John and Laura Ruetsche (pre-print), "Understanding the Role of Unitarily Inequivalent Representations in Quantum Theory," .

Fleming, Gordon (2000), "Reeh-Schlieder Meets Newton-Wigner," Philosophy of Science (Proceedings) 67:S495-S515.

Fraser, Doreen (2006), Haag's Theorem and the Interpretation of Quantum Field Theories with Interactions, University of Pittsburgh: unpublished dissertation.

Fraser, Doreen (2008), "The fate of 'particles' in quantum field theories with interactions," Studies in History and Philosophy of Modern Physics 39:841-859.

Fraser, Doreen (2009), "Quantum field theory: Underdetermination, inconsistency, and idealization," Philosophy of Science 76:536-567.

Fraser, Doreen (2011), "How to take particle physics seriously: A further defence of axiomatic quantum field theory," Studies in the History and Philosophy of Modern Physics 42:126-135.

Greaves, Hilary (2010), "Towards a geometrical understanding of the CPT theorem," British Journal for the Philosophy of Science 61:27-50.

Greaves, Hilary and Teruji Thomas (2014), "On the CPT theorem," Studies in History and Philosophy of Modern Physics 45:46-65.

Halvorson, Hans (2001), "Reeh-Schlieder defeats Newton-Wigner: On alternative localization schemes in relativistic quantum field theory," Philosophy of Science 68:111-133.

Halvorson, Hans and Rob Clifton (2001), "Are Rindler quanta real? Inequivalent particle concepts in quantum field theory," British Journal for the Philosophy of Science 52:417-470.

Halvorson, Hans and Rob Clifton (2002), "No Place for Particles in Relativistic Quantum Theories?" Philosophy of Science 69:128.

Huggett, Nick and Robert Weingard (1996), "Critical Review: Paul Teller's Interpretive Introduction to Quantum Field Theory," Philosophy of Science 63:302-314.

Ismael, Jenann and Jonathan Schaffer (forthcoming), "Quantum Holism," Synthese .

Lewis, Peter J. (2013), "Review of David Wallace, The Emergent Multiverse: Quantum Theory according to the Everett Interpretation," Notre Dame Philosophical Reviews .

Malament, David B. (1996), "In Defense of Dogma: Why There Cannot be a Relativistic Quantum Mechanics of (Localizable) Particles," in Clifton, Rob (ed.), Perspectives on Quantum Reality, Dordrecht: Kluwer Academic Publishers, 1-10. 
Rachiele, Joseph (in progress), "On Whether QFT Describes the Natures of Fundamental Entities: A Cautionary Tale for No-Go Results About Ontology," .

Ruetsche, Laura (2002), "Interpreting Quantum Field Theory," Philosophy of Science 69:348-378.

Ruetsche, Laura (2011), Interpreting Quantum Theories, Oxford: Oxford UP.

Summers, Stephen J. (2001), "On the Stone-von Neumann uniqueness theorem and its ramifications," in Redei, M. and M. Stoeltzner (eds.), John von Neumann and the Foundations of Quantum Physics, Netherlands: Kluwer Academic Press, 135-152.

Swanson, Noel W. (preprint), "PCT Symmetry and the Geometry of Quantum Statespace," .

't Hooft, Gerard (2007), "The Conceptual Basis of Quantum Field Theory," in Butterfield, Jeremy and John Earman (eds.), Philosophy of Physics, North-Holland.

Teller, Paul (1995), An Interpretive Introduction to Quantum Field Theory, Princeton, NJ: Princeton UP.

Teller, Paul (2002), "So What Is the Quantum Field?" in Kuhlmann, Meinard, Holger Lyre and Andrew Wayne (eds.), Ontological Aspects of Quantum Field Theory, River Edge, NJ: World Scientific, $145-164$.

van Fraassen, Bas C. (1991), Quantum Mechanics: An Empiricist View, Oxford: Oxford UP.

von Neumann, John (1932), Mathematical Foundations of Quantum Mechanics, Princeton: Princeton UP.

Wald, Robert M. (1994), Quantum Field Theory on Curved Spacetime and Black Hole Thermodynamics, Chicago: University of Chicago Press.

Wallace, David (2006), "In Defence of Naiveté: On the Conceptual Status of Lagrangian Quantum Field Theory," Synthese 151:33-80.

Wallace, David (2011), "Taking particle physics seriously: A critique of the algebraic approach to quantum field theory," Studies in History and Philosophy of Modern Physics 42:116-125.

Wallace, David and Christopher Gordon Timpson (2007), "Non-locality and Gauge Freedom in Deutsch and Hayden's Formulation of Quantum Mechanics," Foundations of Physics 37:951955.

Wallace, David and Christopher Gordon Timpson (2010), "Quantum Mechanics on Spacetime I: Spacetime State Realism," British Journal for the Philosophy of Science 61:697-727. 